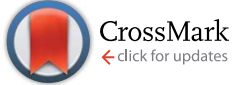

Cite this: RSC Adv., 2017, 7, 396
Received 20th October 2016 Accepted 27th November 2016

DOI: $10.1039 / c 6 r a 25548 \mathrm{e}$

www.rsc.org/advances

\section{Dietary supplementation of Thai black rice bran extract and yeast beta-glucan protects the dextran sodium sulphate mediated colitis induced rat $\uparrow$}

\author{
Noppawat Pengkumsri, ${ }^{\text {ab }}$ Bhagavathi Sundaram Sivamaruthi, ${ }^{a}$ Sasithorn Sirilun, ${ }^{a}$ \\ Prasit Suwannalert, ${ }^{c}$ Teerapat Rodboon, ${ }^{c}$ Chutinun Prasitpuriprecha, ${ }^{d}$ \\ Sartjin Peerajan, ${ }^{e}$ Widawal Butrungrod ${ }^{e}$ and Chaiyavat Chaiyasut ${ }^{\star a}$
}

\begin{abstract}
The present study was employed to evaluate the impact of black rice bran (BRB) extract, and yeast $\beta$-glucan (YBG) supplementation on a dextran sodium sulfate (DSS)-induced colitis rat model. Serum level antioxidant enzymes, cytokines, and histopathological changes were studied by spectrophotometric, ELISA, HPLC, and microscopic analysis. The preventive effect of RB + YBG combinational treatment for DSS-induced colitis in rat was greater than that of RB extract and YBG regarding serum antioxidant level. The elevated expression of the studied inflammatory cytokines (IL-6, IL-17, IFN- $\gamma$ ) was effectively more attenuated by RB + YBG combinational treatment than other tested interventions, which was accompanied by an increase in antiinflammatory cytokines (IL-10, TGF- $\beta$ ). The histological study supported that RB + YBG supplementation improves the health status of DSS-induced colitis rats. The results suggested that the supplementation of $\mathrm{RB}+\mathrm{YBG}$ was a potent alternative nutrient based therapeutic agent for colitis and to prevent the development of cancer.
\end{abstract}

\section{Introduction}

Ulcerative colitis (UC), inflammation in the colon region, is one of the inflammatory bowel diseases (IBD) with unknown etiology. In general, IBD is characterized by the chronic unrestrained inflammation of the gastrointestinal tract and intestinal mucosa. ${ }^{1}$ IBD is closely allied with an increased risk of colon cancer, though it starts with the inflammation of mucosal cells. $^{2}$ The risk of development of colorectal cancer among colitis patients is increased about 10 -fold. ${ }^{3}$ The supplementation of anti-inflammatory mediators helps to suppress the colonic inflammation and risk of UC-associated colon cancer development. ${ }^{4}$ 2,4,6-Trinitrobenzene sulfonic acid (TNBS) and dextran sodium sulphate (DSS) are the two commonly used chemicals to induce IBD in experimental animals. DSS mimics the pathological conditions of UC; DSS with different molecular masses has different effects on the concentration and induction

${ }^{a}$ Department of Pharmaceutical Science, Faculty of Pharmacy, Chiang Mai University, Chiang Mai, Thailand. E-mail: chaiyavat@gmail.com; Tel: +66-539-44340

${ }^{b}$ Faculty of Pharmacy, Huachiew Chalermprakiet University, Samutprakan, Thailand 'Department of Pathobiology, Faculty of Science, Mahidol University, Bangkok, Thailand

${ }^{d}$ Faculty of Pharmaceutical Sciences, Ubon Ratchathani University, Ubon Ratchathani, Thailand

${ }^{e}$ Health Innovation Institute, Chiang Mai, Thailand

$\dagger$ Electronic supplementary information (ESI) available: Table S1, and Fig. S1 and S2. See DOI: $10.1039 / \mathrm{c} 6 \mathrm{ra} 25548 \mathrm{e}$ period. $3 \%$ of DSS ( $5 \mathrm{kDa}$ ) can cause colitis-like disease conditions in a rat model. ${ }^{5}$

The phytochemicals are non-nutrient bioactive plant compounds and recognized as anti-inflammatory agents, which are linked to reduce the risk of several chronic diseases. ${ }^{6}$ Rice is the widely used phytochemicals rich food that accounts for the phenolic compounds, flavonoids, $\beta$-sitosterol, tocotrienols/ tocopherols, $\gamma$-oryzanol, and phytic acid. ${ }^{7}$ The unusual rice varieties are dark in color (black, purple, red, pink, and brown) due to the deposition of pigments in the rice coat. ${ }^{8}$ Thai rice varieties are reported for their nourished phytochemical content, especially black rice. ${ }^{\mathbf{9}, 10}$ Several studies revealed that the rice and rice bran extracts exhibited numerous desirable biological properties like anti-oxidation, ${ }^{\mathbf{1 1}}$ anti-inflammatory effects, ${ }^{12}$ and anti-cancer effect. ${ }^{13}$

The immune activators are involved in the treatment and prevention of diseases. Beta-glucan is a biopolymer of glucose and well recognized immune modulatory agent, which activates the immune response practiced with various therapeutic agents to improve the effects. ${ }^{14}$ Many kinds of $\beta$-glucans are existing in nature and are commonly found in baker's and brewer's yeast, and certain mushrooms, molds, algae and in the bran of oats and barley. The $\beta$-glucans has several beneficial properties and widely used in human and veterinary medicine, cosmetic, pharmaceutical, and chemical industries along with food production. ${ }^{15} \beta$-Glucan of Candida albicans displayed the most potent anti-inflammatory effect in the DSS-induced colitis model. ${ }^{16}$ 
As per our knowledge, no detailed study was carried out on the synergetic effect of black rice bran extract and yeast $\beta$-glucan on colitis in the rat. Thus, the current study was performed to assess the independent, and combined effect of rice bran extract, and YBG on DSS-induced colitis in the rat with respect to the changes in representative cytokines, antioxidant capacity, antioxidant enzyme levels, and pathophysiology of the colon.

\section{Experimental}

\subsection{Black rice bran extraction}

Chiang Mai black rice (Oryza sativa L.) sample was collected from the rice farm at Mae Rim district, Chiang Mai, Thailand and dried at $60{ }^{\circ} \mathrm{C}$ for $48 \mathrm{~h}$. The rice bran (RB) was acquired by milling the fresh rice and separated through a 60-mesh strainer. Rice bran (RB) was extracted using $0.1 \mathrm{~N} \mathrm{HCl}$ in ethanol $(15: 85$ ratio). The polar extracted solution was membrane $(0.45 \mu \mathrm{m})$ filtrated, and evaporated using vacuum evaporator at $40{ }^{\circ} \mathrm{C}$, and stored at $-20^{\circ} \mathrm{C}$ until use.

\subsection{Determination of phenolic acids and anthocyanins by high-performance liquid chromatography}

The RB extract was analyzed for phenolic compounds, and anthocyanins by reversed-phase HPLC with gradient elution as detailed in our previous study. ${ }^{9}$

\subsection{Yeast beta-glucan (YBG) extraction}

Saccharomyces cerevisiae HII31 was obtained from Health Innovation Institute, Chiang Mai and cultured in Yeast ExtractGlucose broth (YG broth) for $48 \mathrm{~h}$ at $30^{\circ} \mathrm{C}$ with the agitation of $200 \mathrm{rpm}$, and cells were harvested by centrifugation at $7500 \mathrm{rpm}, 4^{\circ} \mathrm{C}$ for $10 \mathrm{~min}$. The collected cells were autolyzed in distillation water $\mathrm{pH} 5.0$ at $50^{\circ} \mathrm{C}$ with $120 \mathrm{rpm}$ shaking for $48 \mathrm{~h}$, and the reaction was arrested by incubating at $80^{\circ} \mathrm{C}$ for $15 \mathrm{~min}$. Then the cells were collected by centrifugation, and suspended with 5 fold of $1.0 \mathrm{M}$ sodium hydroxide $(\mathrm{NaOH})$, and stirred at $80{ }^{\circ} \mathrm{C}$ for $2 \mathrm{~h}$. After incubation, suspensions were centrifuged at $6000 \times g, 4{ }^{\circ} \mathrm{C}$ for $25 \mathrm{~min}$. The supernatant was removed, and the pellets were subjected to cleaning and drying process. The cleansing of yeast pellets was done by washing with 3 folds of distillation water and centrifugation at $6000 \times g, 4{ }^{\circ} \mathrm{C}$ for $25 \mathrm{~min}$. The collected pellet was suspended in 5 fold of $1.0 \mathrm{M}$ acetic acid and stirred at $80{ }^{\circ} \mathrm{C}$ for $2 \mathrm{~h}$. After incubation, suspensions were centrifuged at $6000 \times g, 4{ }^{\circ} \mathrm{C}$ for $25 \mathrm{~min}$. The pellet was washed 3 times with distillation water and dried with absolute ethanol using blower hot air oven at $60{ }^{\circ} \mathrm{C}$. The extracted glucan pellets were stored at $4{ }^{\circ} \mathrm{C}$ until analysis. ${ }^{17}$

\subsection{Determination of yeast beta-glucan extract}

The $\beta$-glucan content of the extract was determined by yeast beta-glucan assay kit (Megazyme, Ireland). Briefly, 1,6- $\beta$-Dglucan, $1,3-\beta$-D-glucan, and $\alpha$-glucans are solubilized in $10 \mathrm{~N}$ hydrochloric acid and hydrolyzed by $1.3 \mathrm{~N} \mathrm{HCl}$ at $100^{\circ} \mathrm{C}$ for $2 \mathrm{~h}$. Hydrolysis to D-glucose is facilitated by a mixture of purified exo$1,3-\beta$-glucanase and $\beta$-glucosidase, and the glucose releasing was measured at $510 \mathrm{~nm} .{ }^{17}$

\section{$2.5 \quad$ In vivo study}

About five-weeks-male Wistar rat (an average weight of $170 \mathrm{~g}$ ) were received from National Laboratory Animal Center, Mahidol University, Thailand. The experimental animals were maintained at $25 \pm 2{ }^{\circ} \mathrm{C}$ with $12 \mathrm{~h}$ of the dark-light cycle as per the National Research Council of USA Guideline. The animal experimental protocol was approved by the Ethical Committee for Using and Care of Animals, Faculty of Pharmacy, Chiang Mai University, Thailand (Certificate of ethical clearance no. 01/2015 dated 15 September 2015). The 96 rats were casually separated into 8 groups (12 rats per group) (ESI, Fig. S1 $\dagger$ ), 4 groups of rats were designated as naïve experimental group and another 4 group were designated as colitis induced group. $3 \% \mathrm{w} / \mathrm{v}$ of dextran sodium sulfate (DSS; molecular weight of $5000 \mathrm{Da}$ ) (Wako Co., Ltd., Japan) was prepared in drinking water for the colitis induction. The induction of colitis in the experimental rat was achieved by $3 \%$ DSS, and the colitis condition of DSStreated rats has been already reported. ${ }^{5}$ The DSS was administrated to the respective rats via water for 7 days, and $500 \mathrm{mg}$ $\mathrm{kg}^{-1}$ body weight (BW) of experimental extracts (RB extracts, and $\beta$-glucan) were administrated (oral gavage) for 14 days ( 7 days with DSS +7 days of post-DSS treatment) to the designated rats (ESI, Fig. S1†). The intake of drinking water by each rat was monitored daily by measuring the balance drinking water and calculated by subtracting the weight of the balance drinking water from the initial weight of the water. The weights of experimental rats were recorded carefully. After 14 days, animals were sacrificed and necessary samples (blood, and organs) were collected for the evaluation.

\subsection{Cytokine determination}

The level of selected serum cytokines (IL-6, IL-10, IL-17, INF- $\gamma$, and TGF- $\beta$ ) was determined by ELISA assay kit as per the manufacturer's instructions (R\&D system, USA), and the reaction was recorded by 96-well recorder at $450 \mathrm{~nm}$ (Beckman Coulter DTX 800 multimode detector, USA).

\subsection{Total antioxidant capacity determination}

The 2,2'-azino-bis-3-ethylbenzthiazoline-6-sulphonic acid (ABTS) assay was performed to determine the total antioxidant capacity (TAC) as described. ${ }^{9}$ The results are expressed as $\mathrm{mg}$ trolox equivalents antioxidant capacity (TEAC) per $\mathrm{mL}$ of serum. All samples were tested in triplicate.

\subsection{Determination of malondialdehyde, catalase, superoxide dismutase, and glutathione peroxidase}

The serum malondialdehyde, ${ }^{18}$ catalase, superoxide dismutase, and glutathione peroxidase values were assessed by the modified methods of previous report. ${ }^{19}$ All the experiments were performed in triplicates.

\subsection{Histopathological changes}

The histopathological changes in proximal and distal parts of the colon have been studied as detailed in our previous report. ${ }^{5}$ Hematoxylin and eosin stained colon sections were 
microscopically examined for the pathological signs. The pathological findings of the loss of epithelium and mucosal architecture, infiltration of mononuclear and polymorphonuclear cells in lamina propria, infiltration of inflammatory cells in the submucosa, cryptitis, and abscess formation, and goblet cell depletion were scored. The scoring of selected criterion was graded by 4 levels $(0=$ normal, $1=$ mild, $2=$ moderate, 3 = severe) (ESI, Table S1 ). $^{\circ}$.

\subsection{Statistical analysis}

The antioxidant assays and cytokines level were represented in mean \pm SD. Analysis of variance (ANOVA) was used to test the differences among the values. Least Significant Difference (LSD) test was used to determine the significant differences considering a level of significance at 95\% confident intervals $(p<0.05)$ using the statistical SPSS software version 17.

\section{Results and discussion}

\subsection{Extractions and analysis}

The total phenolic acid content of the black RB (BRB) extract was $252.04 \pm 33.48 \mathrm{mg} \mathrm{g}^{-1}$ of extract (gallic acid equivalent). The major phenolic acids such as protocatechuic acid, caffeic acid, syringic acid, and $p$-coumaric acid were also measured, and the concentration of analyzed phenolic acids in the BRB extract was $0.92 \pm 0.06,1.08 \pm 0.07,0.22 \pm 0.03$ and $11.98 \pm$ $0.69 \mathrm{mg} \mathrm{g}^{-1}$ of extract, respectively. The major anthocyanins in the bran extract of Thai black rice varieties were reported as 3-Oglucosides of cyanidin and peonidin. ${ }^{20}$ In the present study, the total anthocyanin content of the RB extract was 865.21 \pm $42.76 \mathrm{mg} \mathrm{g}^{-1}$ of extract (cyanidin chloride equivalent). In detail, cyanidin 3-glucoside, peonidin 3-glucoside, and cyanidin content was $5.97 \pm 0.39,12.03 \pm 0.68$, and $13.23 \pm 0.72 \mathrm{mg} \mathrm{g}^{-1}$ of extract, respectively (Table 1). The YBG was extracted and examined for the quality. About $930 \pm 30$, and $30 \mathrm{mg} \mathrm{g}^{-1}$ of extract was $\beta$-glucan, and $\alpha$-glucan, respectively (ESI, Fig. S2 $\dagger$ ). The results suggested that the studied $\mathrm{RB}$ extract was rich in phenolic acids, and anthocyanins and the YBG extract contain about $93 \%$ of $\beta$-glucan.

Table 1 The phenolic compounds and anthocyanins in BRB extract

\begin{tabular}{|c|c|c|}
\hline S. no & Compounds & $\begin{array}{l}\mathrm{mg} \text { content/g of extract } \\
(\text { mean } \pm \mathrm{SD})\end{array}$ \\
\hline 1 & Total phenolic acids $(\mathrm{GAE})^{a}$ & $252.04 \pm 33.48$ \\
\hline 2 & Protocatechuic acid & $0.92 \pm 0.06$ \\
\hline 3 & Caffeic acid & $1.08 \pm 0.07$ \\
\hline 4 & Syringic acid & $0.22 \pm 0.03$ \\
\hline 5 & $p$-Coumaric acid & $11.98 \pm 0.69$ \\
\hline 6 & Total anthocyanin content $(\mathrm{CE})^{b}$ & $865.21 \pm 42.76$ \\
\hline 7 & Cyanidin 3-glucoside & $5.97 \pm 0.39$ \\
\hline 8 & Peonidin 3-glucoside & $12.03 \pm 0.68$ \\
\hline 9 & Cyanidin & $13.23 \pm 0.72$ \\
\hline
\end{tabular}

\subsection{Induction of colitis}

To check the consistency in the DSS intake by the experimental rats, the weight of the balance drinking water was measured every day and calculated the significance between individual animals. The results indicated that all the animals have received the relatively equal amount of DSS since no significant changes were observed (Fig. 1A). The reduction in the weight of the DSSinduced colitis group indicates the pathological condition of the rat. The test supplements effectively retain the body mass of the rats, which revealed that the studied supplements are good enough to recover the affected host. In particular, RB + YBG combinational treatment provided the superior protection from the weight loss (Fig. 1B).

\subsection{Serum antioxidant analysis}

The total antioxidant capacity of the experimental rats was represented as trolox equivalents antioxidant capacity (TEAC). There were no significant changes between naïve control (G1) and YBG treated (G3) groups, whereas significance was observed among naïve control (G1), RB-treated groups (G2), and YGB treated group (G3). The DSS-induced colitis control (G5) group showed reduced level of TEAC $(0.48 \pm 0.02 \mathrm{mM})$, whereas treated groups displayed an increase in TEAC, especially RB + YBG treatment (G8) $(0.71 \pm 0.04 \mathrm{mM})$.

Oxidative stress is one of the main factor involved in the carcinogenesis. It is well known that the consumption of red wine, strawberries, vitamin $\mathrm{C}$, and combinations of vegetables increased the plasma antioxidant level of elder people. ${ }^{21,22}$ Moreover, the slight modifications in lifestyle could prevent the cancer development. Phytochemicals of green tea, milk thistle
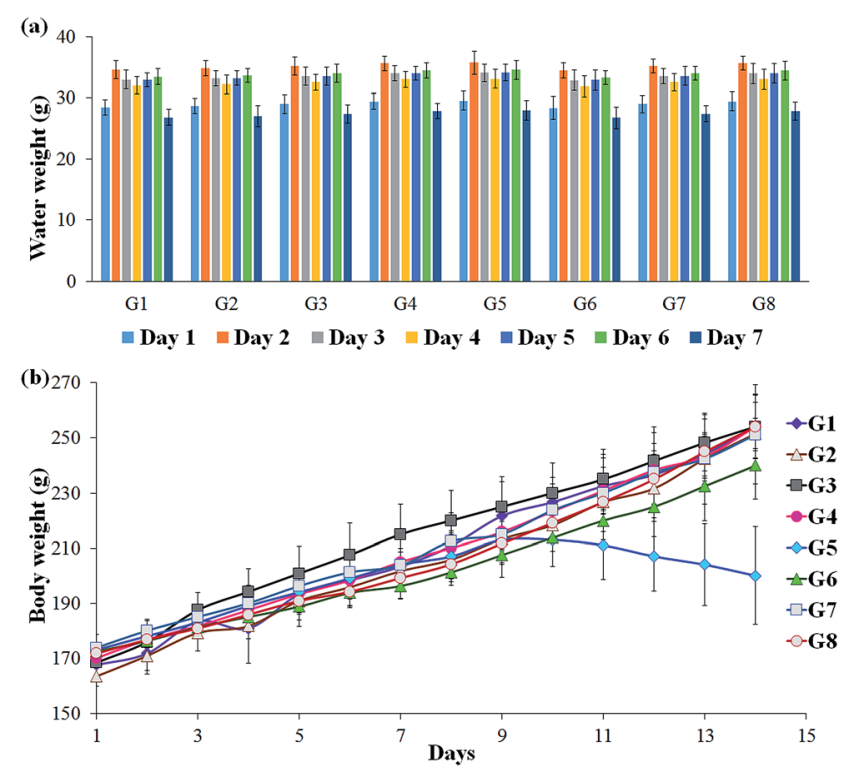

Fig. 1 (a) Weight of the balance drinking water (g) measured every day of DSS mediated induction of colitis in the experimental rat. There was no significant difference among the respective groups. (b) Changes in the body mass $(\mathrm{g})$ of the experimental rats and found that there was no significant changes among the groups except DSS-induced colitis group (G5). 
and grape seeds were reported as potent candidates to prevent the cancer development. ${ }^{23}$ Sreelatha et al. demonstrated that the ethanol extract of Amaranthus paniculatus exhibited anti-tumor property, which was attributed to the antioxidant property of the extract. ${ }^{24}$ Few studies have reported the antioxidant capacity of the black rice bran cultivars of Thailand. ${ }^{10,20}$ A study have revealed that pre-treatment of Lacto-Wolfberry, which is rich in anthocyanins increased the total antioxidant capacity of the plasma and antioxidant biomarkers in the colon of the 2,4,6trinitrobenzene sulfonic acid induced colitic mice. ${ }^{25}$ In a recent study, the anthocyanin-rich wheat based food showed no significant changes in the antioxidant capacity and oxidative stress (advanced oxidation protein products) in the plasma, colon and liver samples of DSS-induced colitic mice. ${ }^{26}$ In the present study, the increase in the antioxidant capacity was higher than the naïve control, which suggested that the supplementation of RB + YBG improved the free radical scavenging ability of the host (Fig. 2a). The serum MDA level was significantly reduced in RB + YBG treatment group (G8) (13.61 \pm $1.91 \mu \mathrm{M}$ ) than that of the DSS-induced colitis control (G5) (27.59 $\pm 1.13 \mu \mathrm{M}$ ) (Fig. 2b), which showed that RB + YBG supplementation increased the lipid peroxidation inhibition ability of the DSS-induced colitis rat. The supplementation of RB extract and YBG increased the catalase level in naïve rats, significantly. Colitis induction drastically reduced the catalase level in G5 $\left(86.60 \pm 10.42 \mathrm{U} \mathrm{mL}^{-1}\right)$, while $\mathrm{RB}$ extract $(201.02 \pm 10.65 \mathrm{U}$

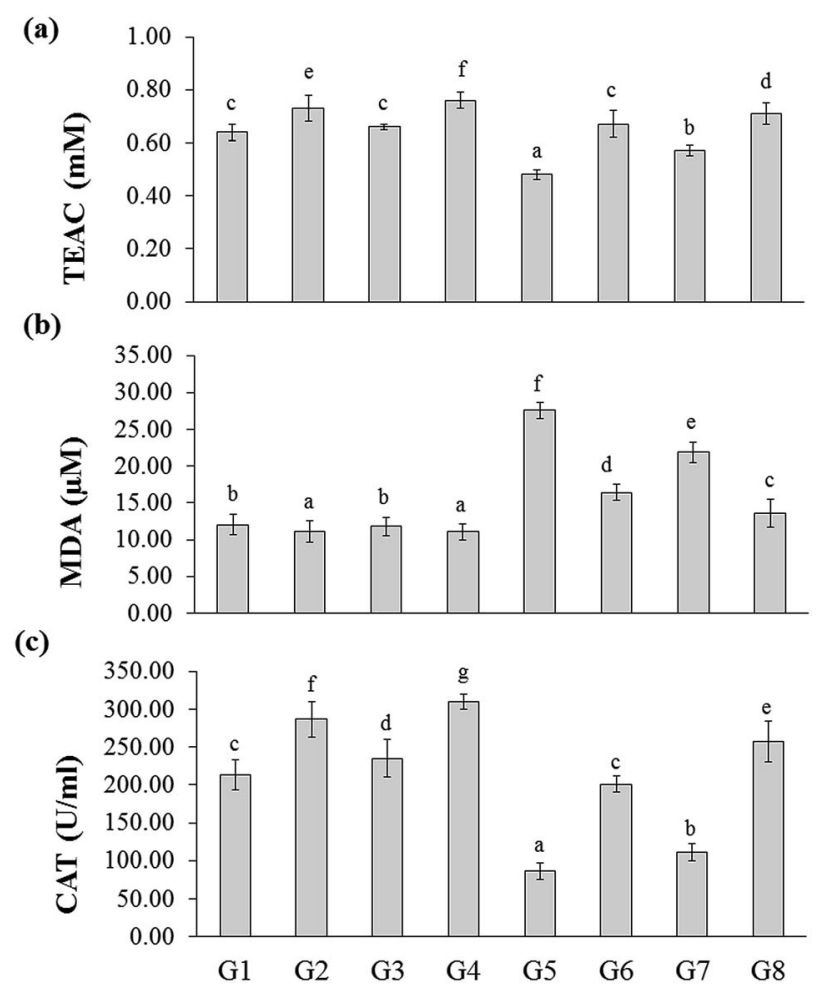

Fig. 2 Effect of supplementation of RB and YBG on the level of (a) total antioxidant capacity (TEAC), (b) lipid peroxidation inhibition ability (MDA), and (c) catalase (CAT) in the serum of the experimental rats. ${ }^{a-g}$ Represents the significant difference between the groups with $p<$ 0.05 .
$\left.\mathrm{mL}^{-1}\right)$, and $\mathrm{RB}+\mathrm{YBG}$ treatment $\left(257.19 \pm 27.19 \mathrm{U} \mathrm{mL}^{-1}\right)$ notably enhanced the level of catalase in G6 and G8 (Fig. 2c). The SOD level in G5 was significantly low than that of the other group due to the colitis condition $\left(50.67 \pm 9.04 \mathrm{U} \mathrm{mL}^{-1}\right)$, but RB + YBG treatment (G8) $\left(98.51 \pm 10.05 \mathrm{U} \mathrm{mL}^{-1}\right)$ bring back the SOD level equal to naïve control (G1) (90.34 $\pm 8.03 \mathrm{U} \mathrm{mL}^{-1}$ ) (Fig. 3a). Likewise, GPx level also improved by the combination of RB + YBG treatment (G8) (237.65 $\left.\pm 16.50 \mathrm{U} \mathrm{mL}^{-1}\right)$, than that of the DSS-induced colitis control group (G5) (183.43 $\pm 12.34 \mathrm{U}$ $\mathrm{mL}^{-1}$ ) (Fig. 3b).

The results suggested that the experimental interventions improved the health of the host. More particularly, RB extract supplementation exhibited the enhanced improvisation of DSSinduced colitis host than that of the YBG, possibly due to the richness of antioxidants in $\mathrm{RB}$ extract. Moreover, combination of $\mathrm{RB}+\mathrm{YBG}$ treatment (G8) improved the antioxidant level of colitis rat, significantly than that of the RB extract (G6), and YBG (G7) supplemented group in all tested parameters (Fig. 2 and 3), which suggested that the intervention of RB + YBG combination nullify the colitis related pathogenic condition by improving the radical scavenging property of the host system.

\subsection{Changes in cytokine level}

In the present study, the inflammatory cytokines (IL-6, IL-17, and IFN- $\gamma$ ) level was elevated in DSS-induced colitis group (G5) $(223.03 \pm 16.51,116.46 \pm 7.23$, and $141.81 \pm 10.67 \mathrm{pg}$ $\mathrm{mL}^{-1}$, respectively) than that of the naïve control group (G1) $\left(164.53 \pm 15.39,86.44 \pm 8.44\right.$, and $91.69 \pm 10.30 \mathrm{pg} \mathrm{mL}{ }^{-1}$, respectively). Whereas RB extract, YBG, and RB + YBG treatment reduced the serum IL-6, IL-17, and IFN- $\gamma$ level significantly (Fig. 4) when compared to that of the DSS-induced colitis group
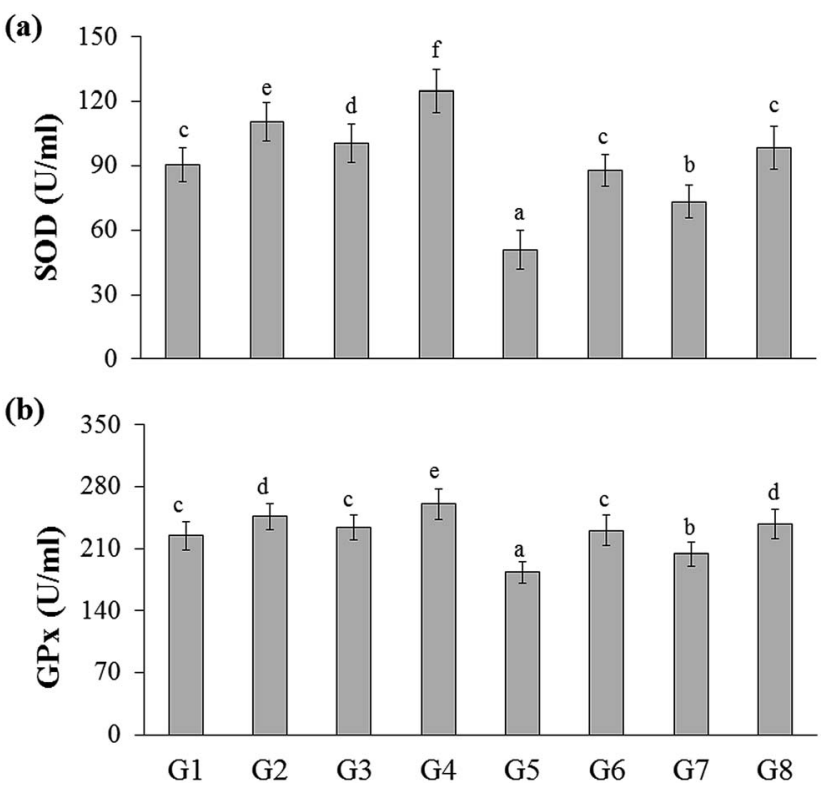

Fig. 3 Effect of supplementation of RB and YBG on the level of (a) superoxide dismutase (SOD) and (b) glutathione peroxidase (GPx) in the serum of the experimental rats. ${ }^{a-g}$ Represents the significant difference between the groups with $p<0.05$. 
(a)

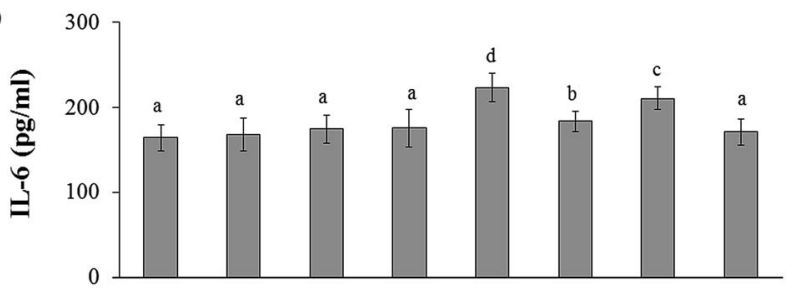

(b)

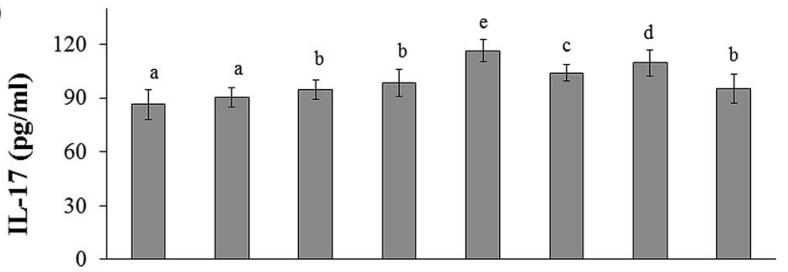

(c)

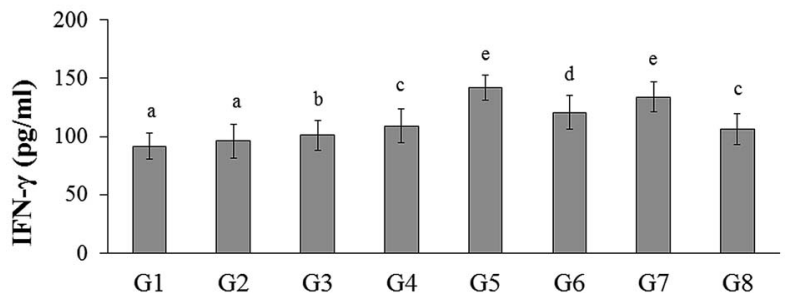

Fig. 4 Effect of supplementation of RB and YBG on the level of (a) IL-6, (b) IL-17, and (c) IFN- $\gamma$ in the serum of the experimental rats. $a-g$ Represents the significant difference between the groups with $p<0.05$

(G5). Notably, the level of IL-6 in DSS-induced colitis rat (G8) $\left(171.18 \pm 15.34 \mathrm{pg} \mathrm{mL}^{-1}\right)$ was reduced to normal range upon RB + YBG treatment (Fig. 4A). Since the administration of betaglucan (microbe associated molecular pattern) might have activated the immune system which leads to inflammation, the level of inflammatory markers (IL-17 and IFN- $\gamma$ ) was increased in the naïve rat supplemented with YBG (G3) and naïve rat supplemented with RB, YBG (G4) when compared to the naïve control group (G1) and naïve rat supplemented with RB (G2).

The anti-inflammatory cytokines are involved in the inflammatory regulation to maintain the effective defense system. The level of representative anti-inflammatory cytokines (IL-10, and TGF- $\beta$ ) was assessed in experimental rats. DSS mediated induction of colitis (G5) suppress the level of IL-10, and TGF- $\beta\left(131.01 \pm 12.10,128.60 \pm 10.18 \mathrm{pg} \mathrm{mL}^{-1}\right.$, respectively) than that of the naïve control $(193.55 \pm 15.94,164.35 \pm$ $12.31 \mathrm{pg} \mathrm{mL}^{-1}$, respectively), while RB + YBG treatment evoked the level of IL-10, and TGF- $\beta$ in DSS-induced colitis rat (G8) $\left(195.64 \pm 14.61,172.88 \pm 11.26 \mathrm{pg} \mathrm{mL}^{-1}\right)$ (Fig. 5A and B). Obviously, YBG supplementation (G3), and RB + YBG combination in naïve rat (G4) elevated the level of cytokines than that of the RB extract (G2), whereas in DSS-induced colitis rat model, RB extract supplemented group (G6) displayed the lower, and higher level of inflammatory, and anti-inflammatory cytokines, respectively (Fig. 4 and 5).

It is known that the germinated brown rice reduced the level of iNOS, activated the p38-MAPK, ERK, NF- $k$ B proteins and COX-2 proteins in DSS-induced colitis mice, and also suppressed the expression of TNF- $\alpha$ and $\mathrm{IL}-6 .{ }^{27}$ The

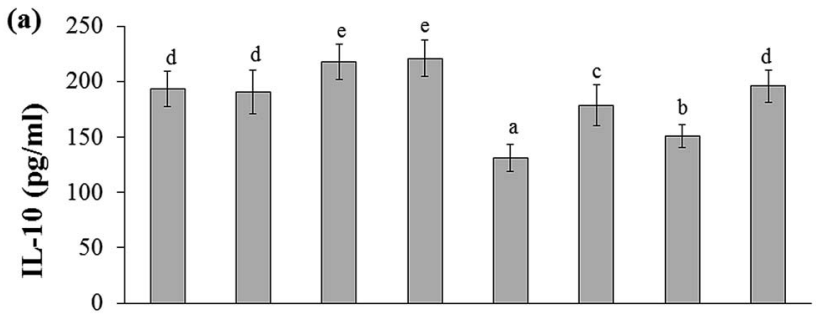

(b)

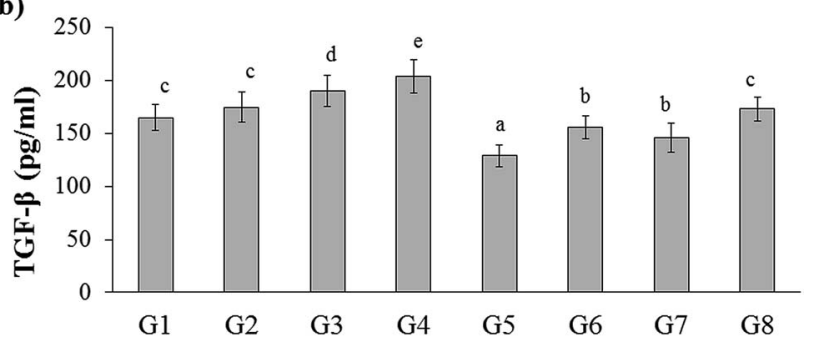

Fig. 5 Effect of supplementation of RB and YBG on the level of (a) IL10 , and (b) TGF- $\beta$ in the serum of the experimental rats. ${ }^{a-g}$ Represents the significant difference between the groups with $p<0.05$.

supplementation of fermented brown rice reduced the colitis index, myeloperoxidase (MPO) activity, and regulates the colonic microbiota. ${ }^{28}$ Rice prolamin extract reduced the level of cleaved caspase-3, number of PCNA-positive cells in colon, and prevent the NF- $\mathrm{\kappa B}$ activation, apoptosis and cell proliferation in DSS induced colitis mice. ${ }^{29}$ da $\mathrm{S}$ Nascimento Santos et al. reported the protective effect of $\beta$-glucan (BG) of Caripia montagnei mushroom. C. montagnei BG reduced the colic lesions, and IL-6, moreover, the level of alkaline phosphatase and myeloperoxidase was also recorded with reduction in cellular infiltration. ${ }^{30} \mathrm{BG}$ from Pleurotus ostreatus protected the rat from colitis possibly by enhancing the antioxidant resistance of the colonic wall against inflammatory violence. ${ }^{31}$ The results of the present study suggested that RB extract was more protective than YBG, regarding cytokine regulation, and the combination of RB + YBG supplementation is the possible way to advance the treatment (Fig. 4 and 5).

\subsection{Histopathological changes}

The impact of test supplements on colon tissues of DSS-induced colitis rats was evaluated by hematoxylin and eosin staining method (Fig. 6A). Both proximal and distal regions of the colon were examined for the prescribed parameters to measure the protective nature of test extracts.

The initial inflammatory phenomena were also reflected in the histopathological analysis. The histological scores were calculated and found that DSS-induced colitis group secured high histological score in the proximal and distal region (5.25, and 15.50, respectively), whereas RB + YBG combinational treatment reverts the adverse condition of the colon $(0.00$, and 2.99, respectively) (Fig. 6B and C). Anthocyanins extract from blueberry significantly prohibited the weight loss and reestablished IL-10 secretion, and reduced the levels of NO, MPO, IL-12, TNF- $\alpha$, and IFN- $\gamma$ in trinitrobenzene sulfonic acid- 
(a)

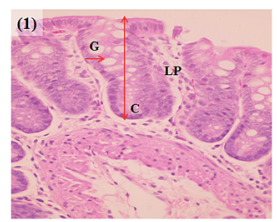

(b)

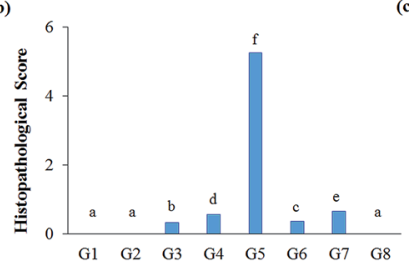

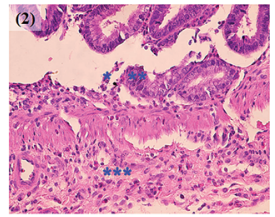

(c)

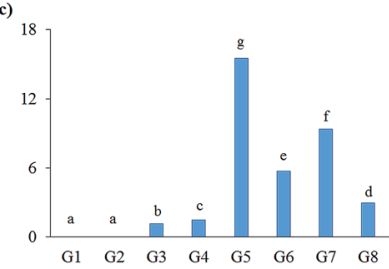

Fig. 6 The distal region of colon histogram (original magnification $400 \times$ ) of naïve control (a-1), DSS-induced colitis (a-2), and RB + YBG treated group (a-3). Goblet cell (G), colonic crypt (C), and lamina propria (LP) were indicated. $*, * *, * * *$ Represents the loss of mucosal architecture, goblet cell depletion, and infiltration of inflammatory cells, respectively. Total pathological score of proximal (b) and distal (c) part of the colon of the experimental rats.

induced mice. ${ }^{32}$ Several fruits anthocyanins were reported for the protective nature of colitis condition. ${ }^{33}$ The present study also supported the curing ability of rice anthocyanins against colitis in DSS-induced colitis rat model. The histopathological study revealed that the supplementation of RB + YBG combination cures the colitis condition (Fig. 6).

\section{Conclusion}

The preventive effect of $\mathrm{RB}+\mathrm{YBG}$ combinational treatment (G8) on DSS-induced colitis rat was greater to that of RB extract (G6), and YBG (G7) regarding serum antioxidant level. The elevated expression of studied inflammatory cytokine (IL-6, IL17 , and IFN- $\gamma$ ) was effectively attenuated by RB + YBG combinational treatment than that of the other tested interventions, which was accompanied by an increase in anti-inflammatory cytokines (IL-10, and TGF- $\beta$ ). This is the primary study revealing the synergetic effect of $\mathrm{RB}$ extract and YBG against colitis in rat, moreover RB + YBG combinational treatment as a potent alternative nutrient based therapeutic agent for colitis, and aid to prevent the subsequent development of colon cancer.

\section{Acknowledgements}

We gratefully acknowledge the National Research University Chiang Mai University (NRU - CMU) and National Research University - Office of Higher Education Commission (NRU OHEC), Ministry of Education, Thailand for the financial supports. The authors also acknowledge the Chiang Mai University Research Grant, Chiang Mai, Thailand. We wish to acknowledge the Faculty of Pharmacy and Chiang Mai University, Chiang Mai, Thailand for the necessary provision. BSS wish to thank CMU-Post Doctoral Fellowship for the support.

\section{References}

1 A. Kaser, S. Zeissig and R. S. Blumberg, Inflammatory Bowel Disease, Annu. Rev. Immunol., 2010, 28, 573-621.

2 S. Danese, Immune and non-immune components orchestrate the pathogenesis of inflammatory bowel disease, Am. J. Physiol.: Gastrointest. Liver Physiol., 2011, 300(5), G716-G722.

3 D. N. Seril, J. Liao, G. Y. Yang and C. S. Yang, Oxidative stress and ulcerative colitis-associated carcinogenesis: studies in humans and animal models, Carcinogenesis, 2003, 24(3), 353-362.

4 D. T. Rubin, A. LoSavio, N. Yadron, D. Huo and S. B. Hanauer, Aminosalicylate therapy in the prevention of dysplasia and colorectal cancer in ulcerative colitis, Clin. Gastroenterol. Hepatol., 2006, 4(11), 1346-1350.

5 N. Pengkumsri, P. Suwannalert, B. S. Sivamaruthi, R. Wongpoomchai, S. Sirisattha, A. Tammasakchai, et al., Molecular, Histological, and Anti-oxidant Evaluation of Colitis Induction in Rats by Different Concentration of Dextran Sodium Sulfate (5 KDA), Int. J. Pharm. Pharm. Sci., 2015, 7(12), 283-287.

6 R. H. Liu, Potential synergy of phytochemicals in cancer prevention: mechanism of action, J. Nutr., 2004, 134(12), 3479S-3485S.

7 A. J. Henderson, C. A. Ollila, A. Kumar, E. C. Borresen, K. Raina, R. Agarwal, et al., Chemopreventive properties of dietary rice bran: current status and future prospects, Adv. Nutr., 2012, 3(5), 643-653.

8 P. Goufo and H. Trindade, Rice antioxidants: phenolic acids, flavonoids, anthocyanins, proanthocyanidins, tocopherols, tocotrienols, $\gamma$-oryzanol, and phytic acid, Food Sci. Nutr., 2014, 2(2), 75-104.

9 N. Pengkumsri, C. Chaiyasut, C. Saenjum, S. Sirilun, S. Peerajan, P. Suwannalert, et al., Physicochemical and antioxidative properties of black, brown and red rice varieties of northern Thailand, Food Sci. Technol., 2015, 35(2), 331-338.

10 N. Pengkumsri, C. Chaiyasut, B. S. Sivamaruthi, C. Saenjum, S. Sirilun, S. Peerajan, et al., The influence of extraction methods on composition and antioxidant properties of rice bran oil, Food Sci. Technol., 2015, 35(3), 493-501.

11 M. Walter, E. Marchesan, P. F. S. Massoni, L. P. da Silva, G. M. S. Sartori and R. B. Ferreira, Antioxidant properties of rice grains with light brown, red and black pericarp colors and the effect of processing, Food Res. Int., 2013, 50(2), 698-703.

12 S. Phutthaphadoong, Y. Yamada, A. Hirata, H. Tomita, A. Hara, P. Limtrakul, et al., Chemopreventive effect of fermented brown rice and rice bran (FBRA) on the inflammation-related colorectal carcinogenesis in ApcMin/ + mice, Oncol. Rep., 2010, 23(1), 53-59.

13 R. Kannappan, V. R. Yadav and B. B. Aggarwal, $\gamma$-Tocotrienol but not $\gamma$-tocopherol blocks STAT3 cell signaling pathway through induction of protein-tyrosine phosphatase SHP-1 and sensitizes tumor cells to chemotherapeutic agents, $J$. Biol. Chem., 2010, 285(43), 33520-33528. 
14 V. Petravic-Tominac, V. Zechner-Krpan, S. Grba, S. Srecec, I. Panjkota-Krbavcic and L. Vidovic, Biological effects of yeast $\beta$-glucans, Agric. Conspec. Sci., 2010, 75(4), 149-158.

15 C. Laroche and P. Michaud, New developments and prospective applications for $\beta(1,3)$ glucans, Recent Pat. Biotechnol., 2007, 1(1), 59-73.

16 S. Jawhara, K. Habib, F. Maggiotto, G. Pignede, P. Vandekerckove, E. Maes, et al., Modulation of intestinal inflammation by yeasts and cell wall extracts: strain dependence and unexpected anti-inflammatory role of glucan fractions, PLoS One, 2012, 7(7), e40648.

17 N. Pengkumsri, B. S. Sivamaruthi, S. Sirilun, S. Peerajan, P. Kesika, K. Chaiyasut, et al., Extraction of $\beta$-glucan from Saccharomyces cerevisiae: comparison of different extraction methods and in vivo assessment of immunomodulatory effect in mice, Food Sci. Technol., 2016, DOI: 10.1590/1678-457X.10716.

18 P. Suwannalert, S. Rattanachitthawat, C. Chaiyasut and S. Riengrojpitak, High levels of 25-hydroxyvitamin D3 [25 $(\mathrm{OH})$ D3] and-tocopherol prevent oxidative stress in rats that consume Thai brown rice, J. Med. Plants Res., 2010, 4, 120-124.

19 W. Luczaj, A. Stankiewicz-Kranc, E. Milewska, W. Roszkowska-Jakimiec and E. Skrzydlewska, Effect of sweet grass extract against oxidative stress in rat liver and serum, Food Chem. Toxicol., 2012, 50(2), 135-140.

20 K. Pitija, M. Nakornriab, T. Sriseadka, A. Vanavichit and S. Wongpornchai, Anthocyanin content and antioxidant capacity in bran extracts of some Thai black rice varieties, Int. J. Food Sci. Technol., 2013, 48, 300-308.

21 G. Cao, R. M. Russell, N. Lischner and R. L. Prior, Serum antioxidant capacity is increased by consumption of strawberries, spinach, red wine or vitamin $\mathrm{C}$ in elderly women, J. Nutr., 1998, 128, 2383-2390.

22 G. Cao, S. L. Booth, J. A. Sadowski and R. L. Prior, Increases in human plasma antioxidant capacity after consumption of controlled diets high in fruit and vegetables, Am. J. Clin. Nutr., 1998, 68, 1081-1087.

23 J. A. Nichols and S. K. Katiyar, Skin photoprotection by natural polyphenols: Anti-inflammatory, antioxidant and DNA repair mechanisms, Arch. Dermatol. Res., 2010, 302, 71-83.

24 S. Sreelatha, E. Dinesh and C. Uma, Antioxidant properties of Rajgira (Amaranthus paniculatus) leaves and potential synergy in chemoprevention, Asian Pac. J. Cancer Prev., 2012, 13, 2775-2780.

25 D. Philippe, V. Brahmbhatt, F. Foata, Y. Saudan, P. Serrant, S. Blum, J. Benyacoub and K. Vidal, Anti-inflammatory effects of Lacto-Wolfberry in a mouse model of experimental colitis, World J. Gastroenterol., 2012, 18(38), 5351-5359.

26 K. Jansakova, J. Babickova, B. Filova, E. Lengyelova, M. Havrlentova, J. Kraic, P. Celec and L. Tothova, Anthocyanin-rich diet in chemically induced colitis in mice, Folia Biol., 2015, 61(3), 104-109.

27 D. K. Park and H. J. Park, Ethanol extract of Antrodia camphorata grown on germinated brown rice suppresses inflammatory responses in mice with acute DSS-induced colitis, J. Evidence-Based Complementary Altern. Med., 2013, 2013, 914524.

28 K. Kataoka, S. Ogasa, T. Kuwahara, Y. Bando, M. Hagiwara, H. Arimochi, et al., Inhibitory effects of fermented brown rice on induction of acute colitis by dextran sulfate sodium in rats, Dig. Dis. Sci., 2008, 53(6), 1601-1608.

29 C. Y. Chung, Y. L. Park, N. Kim, H. H. Oh, D. S. Myung, J. S. Kim, et al., Rice prolamin extract ameliorates acute murine colitis by inhibiting nuclear factor-kappa $\mathrm{B}$ and modulating intestinal apoptosis and cell proliferation, Clin. Exp. Immunol., 2014, 178(3), 537-547.

30 M. da S Nascimento Santos, J. E. de M Magalhães, L. S. E. P. W. Castro, T. de Sousa Pinheiro, D. A. Sabry, L. T. D. B. Nobre, et al., Effect of glucans from Caripia montagnei mushroom on TNBS-induced colitis, Int. J. Mol. Sci., 2014, 15(2), 2368-2385.

31 P. Bobek, V. Nosalova and S. Cerna, Effect of pleuran ( $\beta$ glucan from Pleurotus ostreatus) in diet or drinking fluid on colitis in rats, Nahrung, 2001, 45(5), 360-363.

32 L. H. Wu, Z. L. Xu, D. Dong, S. A. He and H. Yu, Protective effect of anthocyanins extract from blueberry on TNBSinduced IBD model of mice, J. Evidence-Based Complementary Altern. Med., 2011, 2011, 525462.

33 A. Boussenna, J. Cholet, N. Goncalves-Mendes, J. JoubertZakeyh, D. Fraisse, M. P. Vasson, et al., Polyphenol-rich grape pomace extracts protect against dextran sulfate sodium-induced colitis in rats, J. Sci. Food Agric., 2016, 96(4), 1260-1268. 